\title{
Hydrophilic Stars, Amphiphilic Star Block Copolymers, and Miktoarm Stars with Degradable Polycarbonate Cores
}

\author{
${ }_{3}$ Dhanya Augustine, Nikos Hadjichristidis, Yves Gnanou,* and Xiaoshuang Feng* \\ Cite This: https://dx.doi.org/10.1021/acs.macromol.9b02658 \\ Read Online
}

4 ABSTRACT: A facile one-pot synthetic approach toward the 5 realization of star polymers made of degradable carbonate cores is 6 reported. The synthetic strategy involved triethylborane-activated 7 anionic copolymerization of a difunctional epoxide with $\mathrm{CO}_{2}$ 8 initiated by bis(triphenylphosphine)iminium chloride ( $\mathrm{PPNCl}$ ). 9 Vinyl cyclohexene dioxide (VCD) was, thus, used as a difunctional 10 cross-linker, and core compositions with 80-90\% carbonate 11 content were achieved. Poly(ethylene oxide) (PEO) arms were 12 grown from these in-situ generated polycarbonate core anions to 13 build a range of star polymers, including hydrophilic PEO stars and

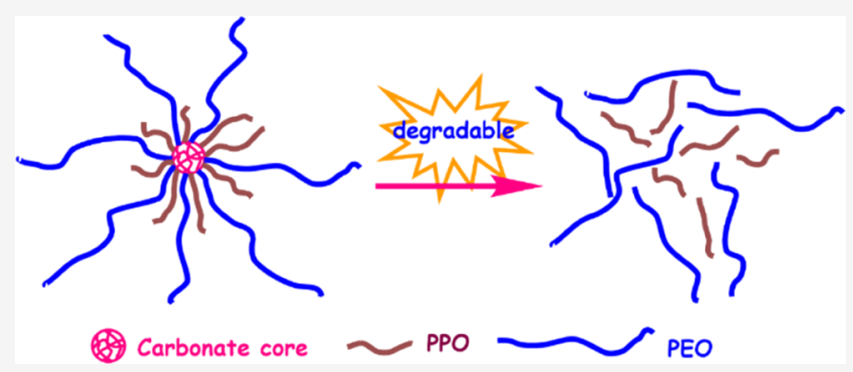
14 star-shaped block copolymers. Poly(propylene oxide) (PPO) precursors were used in a second approach as macroinitiators to form 15 carbonate cores through the arm-first method; miktoarm stars having a large number of arms, both hydrophilic and hydrophobic, 16 could be derived by this method. The carbonate cores of the synthesized stars were readily degraded through hydrolysis of the core 17 carbonate linkages to yield PEO and PPO chains. Potential applications of these types of systems are manifold, especially for PEG18 based drug delivery vehicles.

\section{INTRODUCTION}

20 Poly(ethylene oxide) (PEO), often referred to as poly21 (ethylene glycol), is a widely utilized polymer for the 22 conjugation of biomolecules (PEGylation) for drug delivery 23 due to its unique properties such as chemical stability, 24 hydrophilicity, biocompatibility, and especially "stealth ef25 fect". ${ }^{1-5}$ PEO samples carrying many terminal functional 26 groups are sometimes preferred to mere linear PEO for they 27 offer better protection to conjugated biomolecules and have 28 much higher loading capacities. Therefore, a great deal of effort 29 has been devoted to synthesizing branched PEOs with starlike, 30 dendrimer-like, and hyperbranched structures ${ }^{6-10}$ or linear 31 PEOs with multifunctionalities. ${ }^{6,11}$

32 In comparison to hyperbranched PEOs, usually character33 ized by a broad polydispersity and ill-defined structure, ${ }^{10,12}$ 34 PEO dendrimers exhibit well-defined structures but necessitate 35 tedious multistep synthesis; ${ }^{13-19}$ in contrast PEO-based star 36 polymers have attracted significant attention as their synthetic 37 methodology is relatively easy and cost-effective. ${ }^{20-29}$ Gen38 erally, star-branched PEO structures are composed of a core 39 and radiating arms. PEO stars can be obtained either by linking 40 a given number of linear chains to a central core following the 41 "arm-first" method or by growing branches from a multifunc42 tional initiator via the "core-first" approach, which was well 43 reviewed by Lapienis. ${ }^{8}$ Due to the steric hindrance during the 44 coupling, starlike PEOs synthesized by the "arm-first" method 45 exhibit generally low molar masses; in contrast, PEO stars 46 synthesized by the "core-first" method can be of high molar 47 masses. Although a core that carries a definite number of functional groups affords an accurate number of arms of PEOs, 48 this number of arms is generally very limited by the availability 49 of core molecules of high functionality. ${ }^{24-26}$ When synthesiz- 50 ing PEO stars with a higher number of arms by the "core-first" 51 method, the precursors serving subsequently as the star cores 52 are generally generated through anionic polymerization of 53 cross-linkers, such as divinylbenzene, ${ }^{29}$ diepoxides, or 54 glycidol. $^{20-23,27,30}$ Such PEO stars exhibit special topological 55 features such as a fluctuation in their degree of branching, 56 which can be large, a low solution viscosity, and smaller 57 hydrodynamic volumes compared to their linear ana- 58 logues. $8,31-33$

As a vehicle for the delivery of biomolecules that requires a 60 long residence time in the body, the hydrodynamic size of 61 conjugates after PEGylation should be above $6-8 \mathrm{~nm}$, which is 62 the threshold for glomerular filtration to avoid renal clearance. 63 However, due to their nondegradability, PEOs with molar 64 mass higher than $40 \mathrm{~kg} / \mathrm{mol}$ can accumulate in the body. ${ }^{4,34}$ A 65 number of synthetic ways have been contemplated toward the 66 preparation of degradable linear PEOs: through modification 67 of commercially available PEOs, by polycondensation of PEG 68

Received: December 16, 2019

Revised: January 19, 2020 
Scheme 1. Synthesis of PEO Stars and PEO-b-PPO Star Block Copolymers with a Degradable PVCDC Carbonate Core by the "Core-First" Method

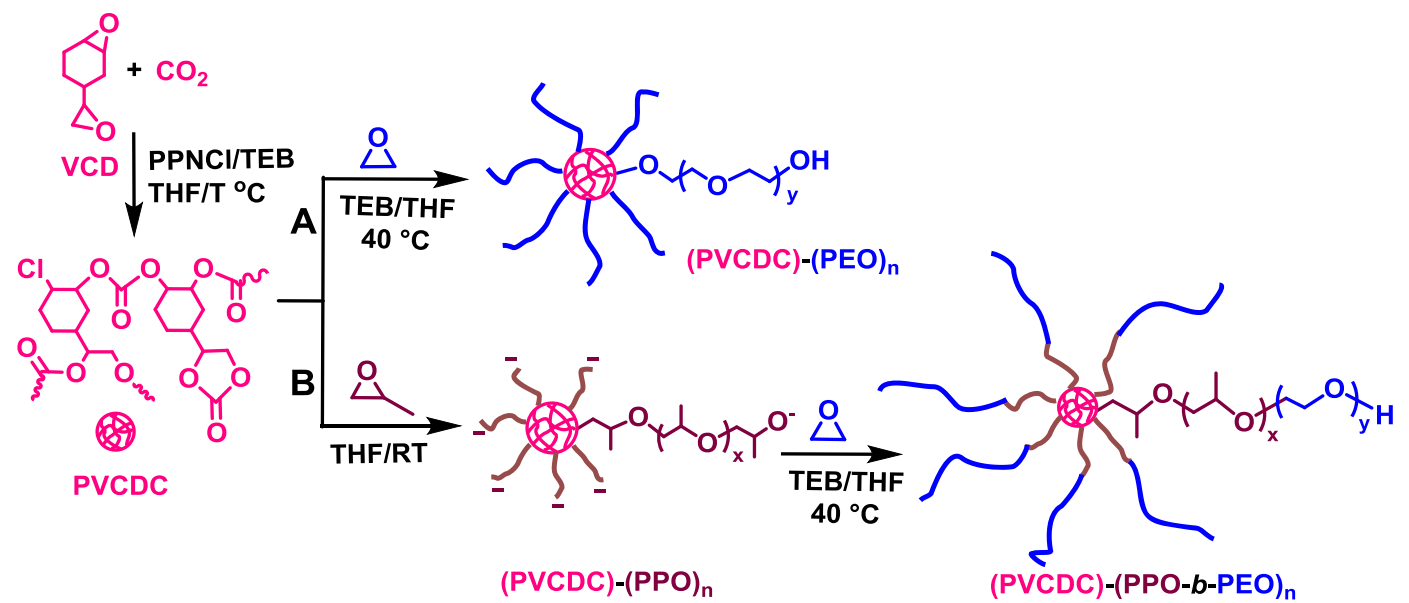

Scheme 2. Synthesis of Miktoarm Stars $\mu$-(PEO $)_{m}(\mathrm{PPO})_{n}$ with Degradable PVCDC Cores

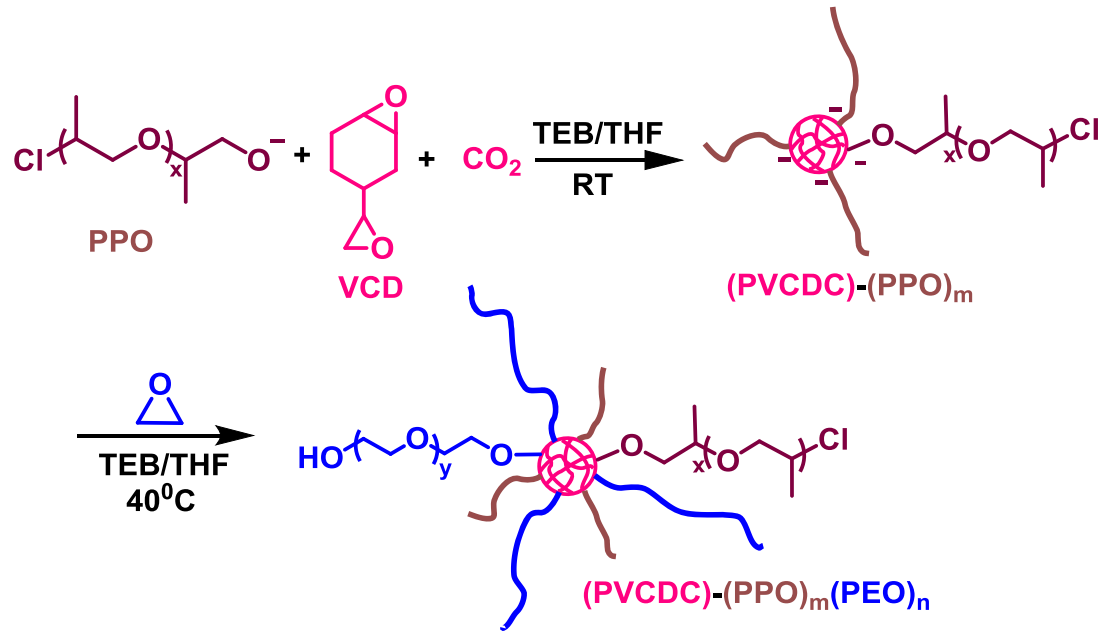

69 telechelics or anionic copolymerization of EO with other 70 monomers to incorporate cleavable moieties, ${ }^{35}$ etc. These 71 attempts were well summarized by Dingels and Frey. ${ }^{36}$ As for 72 star or branched PEOs that can undergo degradation under a 73 trigger, the reports of their synthesis are very few. To be 74 compatible with the anionic ring-opening polymerization 75 (AROP) of EO, which occurs under basic conditions, the 76 strategies used have been to incorporate acid-labile ketal 77 linkages within the core or within branching points by the 78 "core-first" methodology. For instance, we previously reported 79 degradable dendrimer-like PEOs using an original ABC-type 80 branching agent featuring a cleavable ketal group, following an 81 iterative divergent approach based on the AROP of ethylene 82 oxide and the "arborization" of PEO chain ends. ${ }^{14}$ Tonhauser 83 and co-workers designed acid-labile PEO stars by incorporat84 ing acetal moieties into the core structure using an acetal85 containing inimer. ${ }^{37}$ To avoid incompatibility with the 86 conditions of the AROP of EO, one generally resorts to the 87 "arm-first" methodology for the synthesis of degradable PEO 88 stars. For instance, Matyjaszewski et al. synthesized degradable 89 PEO stars, containing disulfide linkages in their core through 90 "arm-first" atom transfer radical copolymerization (ATRP) of a 91 methacrylated PEO macromonomer with disulfide dimetha92 crylate as the cross-linker. ${ }^{38}$ They also resorted to the "core- first" method to obtain stars grown from modified tannic acid 93 serving as the core. ${ }^{39}$ Johnson and co-workers described the 94 one-pot synthesis of core-photocleavable poly(norbornene)-g- 95 PEO brush-arm stars through ring-opening metathesis 96 polymerization (ROMP) of a norbornene-PEO macro- 97 monomer with a photocleavable bis-norbornene cross-linker. ${ }^{40} 98$ This methodology combines graft-through ROMP and "arm- 99 first” cross-linking to introduce degradable linkages within stars 100 during coupling or cross-linking.

Our group recently proposed a new metal-free approach 102 allowing the successful copolymerization of $\mathrm{CO}_{2}$ and epoxides 103 using triethylborane (TEB)-based ate complexes; ${ }^{41,42}$ upon 104 slightly changing the polymerization conditions, the carbonate 105 content $(50-96 \%)$ in the obtained poly(ether-carbonate)s 106 could be easily tuned. Under the same conditions, epoxides 107 such as ethylene oxide, propylene oxide, or glycidyl azide could 108 be effectively homopolymerized in a "living" manner. ${ }^{43-45}$ The 109 above results demonstrate the stability of carbonate linkages in 110 the presence of ate complexes mediating the AROP of 111 epoxides, in contrast to the known incompatibility of ester or 112 carbonate linkages generally observed in the AROP of epoxides 113 under basic conditions: ${ }^{46}$ such incompatibility generally ends 114 up in the degradation of the latter linkages during synthesis. 115 This prompted us to synthesize PEO stars where the cores 116 
117 would be composed of carbonate linkages that remain stable 118 and tolerant toward the growing active species and could, thus, 119 be cleaved after synthesis under hydrolytic conditions. Indeed, 120 degradation investigations have reported that the carbonate 121 linkages could well be hydrolytically degraded in vitro and 122 biodegraded in vivo through surface erosion. ${ }^{47-50}$ In this work, 123 we report the synthesis of PEO stars made of degradable cores 124 in one pot through a "core-first" approach, as shown in Scheme 125 1. The degradable core was first formed through copoly126 merization of a diepoxide (VCD) with $\mathrm{CO}_{2}$; the PEO stars 127 (PVCDC)-(PEO $)_{n}$ were then obtained in the same reactor 128 following addition of EO through AROP in the presence of 129 TEB (Scheme 1A). Based on the above synthesis, hydrophobic 130 stars (PVCDC)-(PPO) $)_{n}$ and amphiphilic star blocks 131 (PVCDC)-(PPO- $b$-PEO) ${ }_{n}$ (Scheme 1B) were further derived 132 through the aforementioned "core-first" method. In another 133 way, (PCVDC)-(PPO $)_{m}(\mathrm{PEO})_{n}$ miktoarm stars were obtained 134 by the "arm-first" and "in-out" methodology (Scheme 2).

\section{EXPERIMENTAL SECTION}

136 Materials. All reactions were carried out under a dry and oxygen137 free argon atmosphere in a Braun Labmaster glovebox. All chemicals 138 purchased from Aldrich and Acros were used without further 139 purification, unless otherwise specially mentioned. Vinyl cyclohexene 140 dioxide (VCD), propylene oxide (PO), and cyclohexene oxide $141(\mathrm{CHO})$ were distilled under reduced pressure over calcium hydride 142 before use. Ethylene oxide (EO) was purified by stirring over $\mathrm{CaH}_{2}$ 143 for one day and distilled into a flask containing $n$-BuLi and stirred for 144 a couple of hours followed by a further distillation. Bis145 (triphenylphosphoranylidene)ammonium chloride ( $\mathrm{PPNCl}$ ) was 146 recrystallized from a methylene chloride-diethyl ether solvent 147 mixture and dried overnight under vacuum at $50{ }^{\circ} \mathrm{C}$. Triethylborane 148 (TEB, $1 \mathrm{M}$ in THF) was used without further purification. 149 Tetrahydrofuran (THF) was distilled from a sodium/benzophenone 150 mixture before use. $\mathrm{CO}_{2}$ (99.995\%) from Abdullah Hashim Industrial 151 \& Gas Co. was further purified by flowing through a purifier of VICI 152 Co.

153 Characterization. ${ }^{1} \mathrm{H}$ and ${ }^{13} \mathrm{C}$ NMR spectra were recorded on a 154 Bruker AVANCE III- $400 \mathrm{~Hz}$ instrument and chemical shifts are 155 reported in ppm using tetramethylsilane as the internal standard in $156 \mathrm{CDCl}_{3}$ solvent. FT-IR spectra were recorded using a Thermoscientific 157 Nicolet iS10 FT-IR spectrometer. The molar mass $\left(M_{\mathrm{n}}\right)$ and molar 158 mass distribution $(\nexists)$ of linear and star polymers were measured on a 159 GPC instrument VISCOTEK VE2001 equipped with Styragel HR2 $160 \mathrm{THF}(1 \mathrm{~mL} / \mathrm{min})$ as the eluent at $35^{\circ} \mathrm{C}$ and a differential refractive 161 index (RI) detector. Polystryrene and PEO standards were used for 162 calibration of the instrument. The absolute molar mass of star 163 polymers was determined by triple-detection GPC (refractometry, 164 light scattering at $\lambda=670 \mathrm{~nm}$, and viscometry). Refractive index 165 increment $(\mathrm{dn} / \mathrm{dc})$ of star samples in THF was measured with a 166 Brookhaven Instruments differential refractometer. Dynamic light 167 scattering (DLS) measurements were performed using a Malvern 168 Zetasizer Nano ZS instrument equipped with a $632.8 \mathrm{~nm} \mathrm{He}-\mathrm{Ne}$ 169 laser. The measurement angle was $173^{\circ}$. For analysis, the polymer 170 sample solution was prepared in DMF solvent and filtered through a $1710.25 \mu \mathrm{m}$ PTFE filter. The cells were temperature-controlled at $20 \pm$ $1720.1{ }^{\circ} \mathrm{C}$. MALDI-TOF MS experiments were performed on a Bruker 173 Ultrafex III MALDI-TOF mass spectrometer (Bruker Daltonik, 174 Bremen, Germany) using trans-2-[3-(4-tert-butylphenyl)2-methyl-2175 propenylidene]malononitrile (DCTB) as the matrix in THF and $176 \mathrm{NaTFA}$ as the ionizing agent. Typically, $40 \mathrm{mg}$ of DCTB, $10 \mathrm{mg}$ of 177 sample, and $10 \mathrm{mg}$ of NaTFA were dissolved in $1 \mathrm{~mL}$ of THF 178 separately and mixed in the ratio of $20: 10: 5 \mu \mathrm{L} ; 1 \mu \mathrm{L}$ from that 179 mixture was spotted over the plate for measurement.

180 Synthesis of PEO Stars with Polycarbonate Cores (PVCDC)181 (PEO) $)_{n}$. Reactions were carried out in a $100 \mathrm{~mL}$ Parr reactor with an 182 in-built charging port, which was dried at $120^{\circ} \mathrm{C}$ overnight and then 183 evacuated in a glovebox chamber for $3 \mathrm{~h}$. For illustrating the synthetic procedure, the core sample designated as (PVCDC1)-(EO $\left(\mathrm{EO}_{300}\right)_{n}$ in 184 entry 1 in Table 2 is taken as a representative. PPNCl $(0.057 \mathrm{~g}, 0.1185$ $\mathrm{mmol})$ was first added into the reactor, followed by THF $(2.5 \mathrm{~mL}) 186$ and TEB ( $0.1 \mathrm{~mL}, 1$ equiv). To this reaction mixture, vinyl 187 cyclohexene dioxide $(64 \mu \mathrm{L}, 0.5 \mathrm{mmol})$ was introduced, and then 188 the reactor was closed and taken out from the glovebox to charge $\mathrm{CO}_{2} 189$ to 10 bar. The polymerization was carried out at $80^{\circ} \mathrm{C}$ for $15 \mathrm{~h}$. After 190 cooling the reactor and $\mathrm{CO}_{2}$ being slowly released, $\mathrm{EO}(1.3 \mathrm{~mL}, 30191$ $\mathrm{mmol})$ and TEB $(0.3 \mathrm{~mL})$ in THF $(13 \mathrm{~mL})$ were carefully injected 192 into the reactor through the charging port, and polymerization was 193 done at $40{ }^{\circ} \mathrm{C}$ for another $4 \mathrm{~h}$. Finally, the reaction mixture was 194 quenched with $\mathrm{HCl}$ in methanol $(1 \mathrm{~mol} / \mathrm{L})$. The obtained crude 195 product was purified through precipitation in diethyl ether and dried 196 in a vacuum oven at $40{ }^{\circ} \mathrm{C}$ overnight.

197

For the characterization of the core formed, the same 198 copolymerization of VCD with $\mathrm{CO}_{2}$ has to be done as above and 199 then quenched using $\mathrm{HCl}$ in methanol $(1 \mathrm{~mol} / \mathrm{L})$. The obtained 200 crude PVCDC core product was purified through precipitation in 201 methanol and dried under vacuum at $40{ }^{\circ} \mathrm{C}$ overnight.

202

Synthesis of Star Block (PCVDC)-(PPO-b-PEO) $)_{n}$. Entry 1 in 203 Table 3, star block sample (PCVDC1)-( $\left(\mathrm{PO}_{87}-b-\mathrm{EO}_{340}\right)_{n}$, was taken as 204 an example. Polycarbonate core PVCDC1 was synthesized as 205 illustrated in the synthesis of homostars. To the synthesized 206 PVCDC1 core mixture $(0.1 \mathrm{mmol})$ inside an autoclave, PO (1.3 207 $\mathrm{mL}, 8.7 \mathrm{mmol})$ in THF $(2.6 \mathrm{~mL})$ was injected, and the 208 polymerization was carried out at RT under stirring for $6 \mathrm{~h}$. Then, 209 EO $(1.7 \mathrm{~mL}, 34 \mathrm{mmol})$ and TEB $(0.3 \mathrm{~mL})$ in THF $(17 \mathrm{~mL})$ were 210 sequentially injected through the charging port and polymerization 211 was continued at $40{ }^{\circ} \mathrm{C}$ for another $4 \mathrm{~h}$. At the end of polymerization, 212 the reaction mixture was quenched with $\mathrm{HCl}$ in methanol ( $1 \mathrm{~mol} / \mathrm{L}) .213$ The crude product was purified through precipitation in hexane, 214 followed by drying in a vacuum oven at $40{ }^{\circ} \mathrm{C}$ overnight. 215

Synthesis of Miktoarm Star Copolymers (PVCDC)- 216 $(\mathrm{PPO})_{m}(\mathrm{PEO})_{n}$. A typical synthetic procedure of miktoarm star 217 copolymers is described here by taking entry 3 in Table 4 as an 218 example. The poly(propylene oxide) (PPO, $M_{\mathrm{n}}=4.2 \mathrm{~kg} / \mathrm{mol}$ ) 219 macroinitiator was first synthesized in a $100 \mathrm{~mL}$ dried Parr autoclave 220 by stirring $\mathrm{PPNCl}(34 \mathrm{mg}, 0.06 \mathrm{mmol})$, TEB ( $60 \mu \mathrm{L}, 0.06 \mathrm{mmol})$, and 221 PO $(0.27 \mathrm{~mL}, 4.3 \mathrm{mmol})$ in THF $(0.27 \mathrm{~mL})$ at RT for $1 \mathrm{~h}$. After VCD 222 $(183 \mu \mathrm{L}, 1.4 \mathrm{mmol})$ in THF $(6 \mathrm{~mL})$ was injected, the reactor was 223 charged with $\mathrm{CO}_{2}$ to $10 \mathrm{bar}$, and kept at $50{ }^{\circ} \mathrm{C}$ for $17 \mathrm{~h}$ under stirring 224 to form the star macroinitiator. The reactor was then cooled and $\mathrm{CO}_{2} 225$ was slowly released, EO $(1 \mathrm{~mL}, 20 \mathrm{mmol})$ and TEB $(180 \mu \mathrm{L}, 0.18226$ $\mathrm{mmol})$ in THF $(10 \mathrm{~mL})$ were finally charged, and polymerization was 227 carried out at ambient temperature for another $1 \mathrm{~h}$. The polymer- 228 ization was quenched with $\mathrm{HCl}$ in methanol $(1 \mathrm{~mol} / \mathrm{L})$. The product 229 was obtained through precipitation in diethyl ether followed by drying 230 in a vacuum oven at $40{ }^{\circ} \mathrm{C}$ overnight.

Hydrolytic Degradation of Star Polymers. In a typical 232 hydrolysis reaction, $60 \mathrm{mg}$ of star sample was dissolved in $4 \mathrm{~mL}$ of 233 THF, followed by the addition of $2 \mathrm{M} \mathrm{KOH}$ in methanol. The 234 hydrolysis reaction was carried out at $50{ }^{\circ} \mathrm{C}$ under stirring for 3 days 235 and then the solvent was removed under reduced pressure. 236 Dichloromethane was added to extract the polymer. The hydrolyzed 237 polymer samples were obtained after concentration and drying. $\quad 238$

\section{RESULTS AND DISCUSSION}

Synthesis of PEO Stars with a Degradable Polycar- 240 bonate Core. Synthesis of Polycarbonate Cores. PEO stars 241 having a degradable core made of carbonate linkages were first 242 prepared by the "core-first" approach, as shown in Scheme 1. 243 VCD was used as the cross-linker (difunctional monomer) to 244 form the degradable polycarbonate core by copolymerization 245 with $\mathrm{CO}_{2}$ using a boron-based ate complex as the initiator 246 (Scheme 1). ${ }^{42}$ VCD contains two types of epoxides, one 247 alicyclic and disubstituted and the other monosubstituted, both 248 known to exhibit different reactivities; however, no clear 249 chemoselectivity was evidenced since macrogel formation was 250 
Table 1. Results of TEB-Activated Copolymerization of VCD with $\mathrm{CO}_{2}$ under Different Conditions ${ }^{a}$

\begin{tabular}{|c|c|c|c|c|c|c|c|}
\hline entry & samples & {$\left[\mathrm{Cl}^{-}\right]:[\mathrm{TEB}]:[\mathrm{VCD}]$} & $T\left({ }^{\circ} \mathrm{C}\right)$ & conv. $(\%)^{b}$ & carbonate content $(\%)^{c}$ & $M_{\mathrm{n}(\mathrm{GPC})} / \bigoplus(\mathrm{kg} / \mathrm{mol})^{d}$ & $F^{e}$ \\
\hline 1 & PVCDC1 & $1: 1: 5$ & 80 & $>99$ & 85 & $5.9 / 1.8$ & 4.6 \\
\hline 2 & PVCDC2 & $1: 1: 5$ & 70 & $>99$ & 88 & $6.3 / 1.8$ & 6.3 \\
\hline 3 & PVCDC3 & $1: 1: 5$ & 60 & $>99$ & 90 & $8.6 / 5.6$ & 8.5 \\
\hline 4 & PVCDC4 & $1: 1: 5$ & 50 & $>99$ & 90 & $12.0 / 5.3$ & 20.8 \\
\hline 5 & PVCDC5 & $1: 1: 6$ & 80 & $>99$ & 90 & $9.6 / 3.7$ & 9.2 \\
\hline 6 & PVCDC6 & $1: 1: 7$ & 80 & gel & & & \\
\hline 7 & PVCDC7 & $1: 1.5: 5$ & 70 & $>99$ & 88 & $7.0 / 4.1$ & 7.7 \\
\hline 8 & PVCDC8 & $1: 1: 6$ & 60 & gel & & & \\
\hline $9^{f}$ & PVCDC9 & $1: 1: 5$ & 70 & $>99$ & 89 & $6.7 / 1.9$ & 5.8 \\
\hline $10^{g}$ & PVCDC10 & $1: 1: 5$ & 80 & $>99$ & 90 & $7.5 / 2.3$ & 11.8 \\
\hline
\end{tabular}

${ }^{a}$ Copolymerization was carried out for $15 \mathrm{~h}$ with a VCD/THF $(\mathrm{v} / \mathrm{v})$ ratio equal to $1: 2.5 \mathrm{using} \mathrm{PPNCl}$ as the initiator or as otherwise mentioned.

${ }^{b}$ Conversion was measured by ${ }^{1} \mathrm{H}$ NMR of the crude reaction mixture. ${ }^{c}$ Determined by ${ }^{1} \mathrm{H}$ NMR. ${ }^{d}$ Measured by GPC with THF as the eluent and calibrated using polystyrene standards. ${ }^{e}$ The functionality was taken from the arm numbers of PEO stars in Table $2 .{ }^{f} \mathrm{Volume}$ ratio of VCD/THF was $1: 2 .{ }^{g}$ Monofunctional $\mathrm{CHO}$ was added to the cross-linker in the molar ratio of $\mathrm{VCD} / \mathrm{CHO}=1: 1$ and $\mathrm{VCD} / \mathrm{THF}(\mathrm{v} / \mathrm{v})=1: 2.5$.

251 observed even at a moderate concentration of VCD. Soluble 252 polycarbonates were, however, reported upon discontinuing 253 the copolymerization of VCD with $\mathrm{CO}_{2}$ at moderate 254 conversion. $^{52}$ To avoid macrogelation when using the "core255 first" methodology, low amounts of VCD were used with a 256 ratio of VCD to initiator smaller than 5 . The challenge here 257 was indeed to prepare colloidal, submicron size range, cross258 linked polycarbonates and, thus, to avoid macrogelation. The 259 parameters that generally play an essential role in the 260 formation of such colloidal, yet reactive, nanogels, which can 261 eventually serve as cores for star synthesis, are the 262 concentration of the reaction medium and the ratios of 263 cross-linker (VCD) to the initiator and to the comonomer. 264 The temperature $(T)$ of the reaction is another parameter to 265 be considered as a very high temperature can favor the 266 formation of cyclic carbonates and, thus, the generation of 267 unproductive intramolecular carbonate linkages, from the 268 cross-linking standpoint. The polymerization conditions and 269 results are listed in Table 1.

270 As shown previously, ${ }^{42}$ the copolymerization behavior of $271 \mathrm{CO}_{2}$ with propylene oxide (PO) is different from that of 272 alicyclic cyclohexene oxide ( $\mathrm{CHO}$ ) when using onium salts as 273 an initiator in the presence of TEB. In the case of CHO, only 274 alternating copolymers with $\mathrm{CO}_{2}$ (polycarbonates) were 275 obtained with $100 \%$ selectivity of linear vs cyclic carbonates; 276 in the case of PO, a poly(ether-co-carbonate) was formed, 277 containing about $10 \%$ of ether linkages along with some cyclic 278 propylene carbonate side products, whose amount was found 279 to increase with the temperature of polymerization. Unlike 280 poly(ether-co-carbonate)s or linear polycarbonates that do 281 contribute to cross-linking, cyclic carbonates do not participate 282 in cross-linking reactions during the copolymerization of VCD 283 with $\mathrm{CO}_{2}$. Before adding ethylene oxide, the product of the 284 copolymerization of VCD with $\mathrm{CO}_{2}$ was sampled out and 285 thoroughly characterized.

286 As shown in Table 1, a ratio of VCD to initiator equal to 5 287 leads to soluble polycarbonate cores and results in a complete 288 conversion of VCD. The obtained polycarbonate cores exhibit 289 a broad molar mass distribution, probably due to occurrence of 290 core-core coupling reactions (Figure 1A). As shown in a 291 representative NMR spectrum (Figure 2A), the peaks 292 corresponding to the CHO-type epoxide units $(3.2 \mathrm{ppm})$ of 293 VCD and the PO-type epoxide units (2.5 and $2.7 \mathrm{ppm})$ of the 294 same cross-linker have all disappeared, indicating the complete 295 reaction of diepoxides. The peaks corresponding to the acyclic

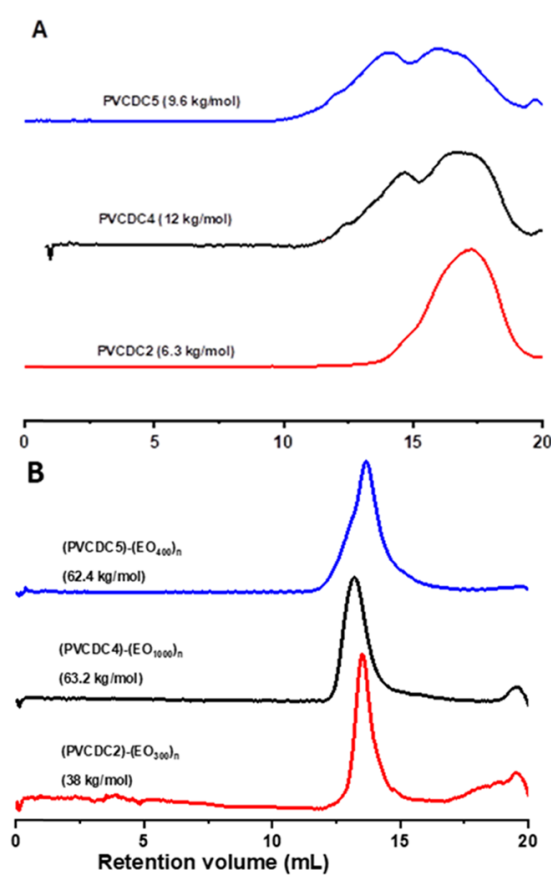

Figure 1. Representative GPC overlay profiles of (A) PVCDC polycarbonate cores and (B) (PVCDC)-(PEO) ${ }_{n}$ stars.

and cyclic carbonate linkages overlap in the region between 5.0296 and $4.0 \mathrm{ppm}$; their presence can also be clearly detected by IR 297 spectroscopy (Figure S1), with strong absorption peaks at 298 1800 and $1740 \mathrm{~cm}^{-1}$ attributable to the stretching of the 299 carbonyl of cyclic and acyclic carbonates, respectively. The 300 selectivity of acyclic vs cyclic carbonates during core synthesis, 301 as determined by IR analysis, falls typically in the range of 63- 302 $68 \%$ except for entry 10, which indicates $80 \%$ selectivity. 303 Besides carbonate linkages, resonances in the region of 3.3-3.5 304 ppm (Figure 2A) show also the presence of ether linkages 305 resulting from the homopolymerization of VCD. Based on the 306 ratio of intensities of the peaks located between 4 and 5 ppm 307 to those of the peaks found around $3.5 \mathrm{ppm}$, the carbonate 308 content of the formed cores could be deduced. As shown in 309 Table 1, the carbonate vs ether content does not change 310 considerably with the temperature and stands at around 90\%. 311 In contrast, the apparent molar masses of the obtained cores 312 increase with the decrease of the polymerization temperature. 313 Since the formation of cyclic carbonate linkages does not 314 

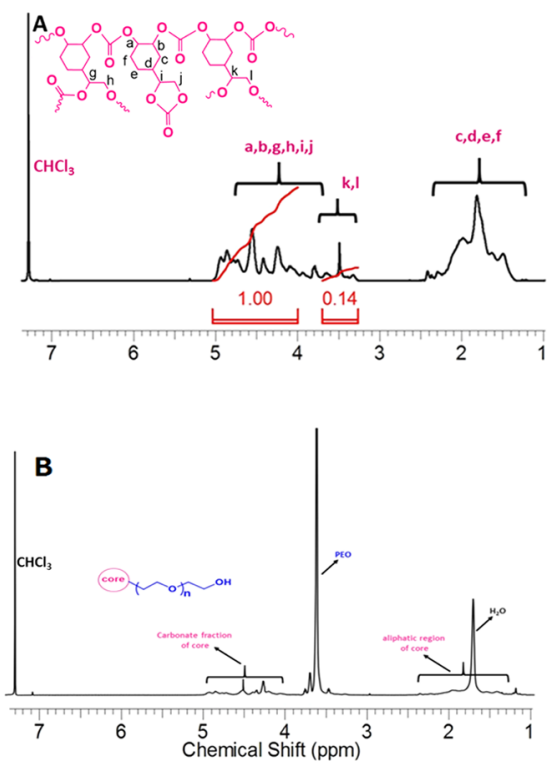

Figure 2. Representative ${ }^{1} \mathrm{H}$ NMR spectra in $\mathrm{CDCl}_{3}$ of (A) polycarbonate cores (PVCDC) and (B) (PVCDC)-(PEO) ${ }_{n}$ stars.

315 contribute to cross-linking, lowering the temperature favors the 316 formation of "linear" acyclic carbonate linkages, resulting in a 317 higher degree of cross-linking, and thus higher apparent molar 318 masses and functionality (vide infra) of the synthesized cores. 319 Indeed, the functionality of the cores increases from 4.6 to 20.8 320 when decreasing the temperature of polymerization from 80 to $32150{ }^{\circ} \mathrm{C}$ for a given feeding ratio (entry $1-4$, Table 1 ). To favor 322 the incorporation of the monosubstituted epoxide carried by $323 \mathrm{VCD}$, attempts to add more TEB to increase the cross-linking 324 density and, in turn, the molar mass and functionality of the 325 core were found successful (entry 7 in Table 1). Utilizing a 326 mono-epoxide $(\mathrm{CHO})$ in addition to $\mathrm{VCD}$ and $\mathrm{CO}_{2}$ was also 327 helpful to enhance the extent of cross-linking without 328 provoking the entire gelation of the reaction medium (entry 32910 in Table 1). On the other hand, an increase in the amount 330 of VCD generally resulted in the gelation of the medium 331 (entries 6, 8 in Table 1). When the ratio of VCD to initiator 332 was $<5$, the functionality of the core could, thus, be fine-tuned 333 by varying the polymerization temperature and the amount of
TEB used, and through the introduction of a mono-epoxide as 334 a third monomer; of the three options, the first one, which is 335 the variation of the temperature, proved the most effective. $\quad 336$

Growth of PEO Arms from "Living" Polycarbonate Cores. 337 The highly branched polycarbonate cores prepared according 338 to the conditions previously described served to initiate the 339 polymerization of EO and grow PEO arms (Scheme 1A). 340 Ethylene oxide, together with THF solvent, was then injected 341 into the same Parr reactor after $\mathrm{CO}_{2}$ was gradually released, 342 and the polymerization of ethylene oxide was subsequently 343 carried out at $40{ }^{\circ} \mathrm{C}$ under stirring. Depending on the cores 344 utilized as macroinitiators and the amount of EO charged, 345 PEO stars exhibiting different arm numbers and lengths could 346 be obtained.

An overlay of FT-IR spectra in Figure S1 clearly shows the 348 presence of the characteristic peaks due to carbonate cores, 349 indicating the integrity of carbonate linkages during the 350 polymerization of EO. Likewise, the NMR characterization 351 shows, in addition to the characteristic peak of PEO at 3.77352 ppm, peaks in the 4.0-5.0 ppm region attributable to $\mathrm{CH}$ and 353 $\mathrm{CH}_{2}$ protons of carbonate linkages, confirming the formation 354 of PEO stars (Figure 2B). After the formation of the PEO 355 stars, the GPC traces remarkably shift to the high molar mass 356 region and exhibit distributions that are symmetrical and 357 narrower with a dispersion around 1.5 in most cases (Figure 358 1B). To calculate the number of arms of each PEO star 359 formed, the actual or absolute molar masses of PEO stars were, 360 thus, measured using light scattering. Then, the polycarbonate 361 core of the stars was completely hydrolyzed, and the molar 362 masses of linear PEO arms were determined by GPC calibrated 363 with $\mathrm{PEO}$ standards. Based on the absolute molar mass values, 364 $M_{\mathrm{w}, \mathrm{star}(\mathrm{LS})}$, the arm numbers could be estimated using the 365 following formula

$$
N_{\mathrm{arm}}=M_{\mathrm{w}, \mathrm{star}(\mathrm{LS})} \times W_{\mathrm{PEO}} / M_{\mathrm{n}(\mathrm{arm})}
$$

where $M_{\mathrm{w}, \mathrm{star}(\mathrm{LS})}, M_{\mathrm{n}(\operatorname{arm})}$, and $W_{\mathrm{PEO}}$ are the absolute molar 368 mass of PEO stars, the molar mass of each arm determined by 369 GPC analysis, and the weight percentage of PEO in the stars, 370 respectively. In Table 2 are listed some of the parameters that 371 t 2 were varied from one experiment to another one, such as the 372 temperature of polymerization, the amount of TEB used, and 373 the ratio of EO to the initiator; under these conditions, PEO 374

Table 2. Characterization of (PVCDC)-(PEO $)_{n}$ Star Polymers Synthesized by the "Core-First" Method ${ }^{a}$

\begin{tabular}{|c|c|c|c|c|c|c|}
\hline entry & star polymer & {$[\mathrm{EO}]:\left[\mathrm{Cl}^{-}\right]$} & $M_{\mathrm{n}, \mathrm{star}} / \bigoplus^{b}(\mathrm{~kg} / \mathrm{mol})$ & $M_{\mathrm{w}, \mathrm{star}(\mathrm{LS})}{ }^{c}(\mathrm{~kg} / \mathrm{mol})$ & $M_{\mathrm{n}, \mathrm{PEO}}{ }^{d}(\mathrm{~kg} / \mathrm{mol})$ & $N_{\mathrm{arm}}{ }^{e}$ \\
\hline 1 & $(\mathrm{PVCDC1})-\left(\mathrm{EO}_{300}\right)_{n}$ & 300 & $45.4 / 1.3$ & 57.2 & 12.6 & 4.6 \\
\hline 2 & $(\mathrm{PVCDC} 2)-\left(\mathrm{EO}_{300}\right)_{n}$ & 300 & $38 / 1.1$ & 77.7 & 12.2 & 6.1 \\
\hline 3 & $(\mathrm{PVCDC} 2)-\left(\mathrm{EO}_{500}\right)_{n}$ & 500 & $41.5 / 1.1$ & 125 & 19.0 & 6.5 \\
\hline 4 & $(\mathrm{PVCDC} 3)-\left(\mathrm{EO}_{50}\right)_{n}$ & 50 & $13.0 / 2.4$ & nd & 2.1 & \\
\hline 5 & $(\mathrm{PVCDC} 3)-\left(\mathrm{EO}_{100}\right)_{n}$ & 100 & $23.4 / 1.6$ & nd & 4.4 & \\
\hline 6 & $(\mathrm{PVCDC} 3)-\left(\mathrm{EO}_{400}\right)_{n}$ & 400 & $48.1 / 1.5$ & 140 & 15.0 & 9.3 \\
\hline 7 & $\left(\right.$ PVCDC4) $-\left(\mathrm{EO}_{1000}\right)_{n}$ & 1000 & $63.2 / 1.3$ & 835 & 40.1 & 20.8 \\
\hline 8 & $(\mathrm{PVCDC5})-\left(\mathrm{EO}_{400}\right)_{n}$ & 400 & $62.4 / 2$ & 151 & 16.3 & 9.2 \\
\hline 9 & $(\mathrm{PVCDC} 7)-\left(\mathrm{EO}_{500}\right)_{n}$ & 500 & $44.4 / 1.6$ & 148 & 19.2 & 7.7 \\
\hline 10 & $\left(\text { PVCDC9)-(EO } \mathrm{EO}_{300}\right)_{n}$ & 300 & $38.8 / 1.4$ & 70.1 & 12.0 & 5.8 \\
\hline 11 & $(\operatorname{PVCDC} 10)-\left(\mathrm{EO}_{100}\right)_{n}$ & 100 & $24.1 / 1.6$ & 50.8 & 4.1 & 12.3 \\
\hline 12 & $(\mathrm{PVCDC} 10)-\left(\mathrm{EO}_{300}\right)_{n}$ & 300 & $34.7 / 1.5$ & 144 & 12.8 & 11.2 \\
\hline
\end{tabular}

${ }^{a}$ Polymerization of EO by the formed PVCDC core anion was carried out by keeping the ratio of TEB to PPNCl as 3:1 and EO in THF (v/v, 1 / 10) at $40{ }^{\circ} \mathrm{C}$ for $4 \mathrm{~h}$, and the conversion was calculated by gravimetry. ${ }^{b}$ Determined by GPC with THF as the eluent based on a polystyrene standard. ${ }^{c}$ Determined by GPC equipped with multiangle light scattering (GPC-MALLS). ${ }^{d}$ PEO arms obtained after degradation were determined by GPC based on a PEO standard. ${ }^{e}$ Calculated based on formula 1. 
Table 3. Characterization of (PVCDC)(PPO-b-PEO $)_{n}$ Star Block Polymers Synthesized by the "Core-First" Method ${ }^{a}$

\begin{tabular}{|c|c|c|c|c|c|c|c|}
\hline \multirow[b]{2}{*}{ entry } & \multirow[b]{2}{*}{ core $^{b}$} & \multirow[b]{2}{*}[\mathrm{PO}]{$:[\mathrm{EO}]:\left[\mathrm{Cl}^{-}\right]$} & \multirow[b]{2}{*}{$(\mathrm{PVCDC})-(\mathrm{PPO})_{n} M_{\mathrm{n}} / \bigoplus^{c}(\mathrm{~kg} / \mathrm{mol})$} & \multicolumn{2}{|c|}{$\begin{array}{l}(\mathrm{PVCDC})-(\mathrm{PPO}-b- \\
\mathrm{PEO})_{n}(\mathrm{~kg} / \mathrm{mol})\end{array}$} & \multirow[b]{2}{*}{ PPO- $b$-PEO $(\mathrm{kg} / \mathrm{mol}) M_{\mathrm{n}}{ }^{e}$} & \multirow[b]{2}{*}{$N_{\mathrm{arm}}^{f}$} \\
\hline & & & & $M_{\mathrm{n}} / \nexists^{c}$ & $M_{\mathrm{w}(\mathrm{LS})}{ }^{d}$ & & \\
\hline 1 & PVCDC1 & $87 / 340 / 1$ & $12.8 / 1.6$ & $23.9 / 1.4$ & 114 & 19.1 & 5.9 \\
\hline 2 & PVCDC2 & $44 / 340 / 1$ & $15.7 / 1.4$ & $31.6 / 2.5$ & 145 & 19.6 & 7.4 \\
\hline 3 & PVCDC3 & $87 / 340 / 1$ & $12.5 / 1.3$ & $43.1 / 2.6$ & 225 & 21.0 & 10.7 \\
\hline 4 & PVCDC4 & $44 / 340 / 1$ & $18.9 / 1.7$ & $50.0 / 1.8$ & 380 & 22.1 & 17.3 \\
\hline 5 & PVCDC5 & $87 / 340 / 1$ & $17.4 / 2.3$ & $40.2 / 2.1$ & 245 & 27.2 & 8.2 \\
\hline
\end{tabular}

${ }^{a}$ Polymerization of PO by PVCDC core anions was carried out by keeping the ratio of TEB to PPNCl as $1: 1$ and PO in THF (v/v, $1 / 2$ ) at RT for 6 $\mathrm{h}$; the sequential polymerization of EO was carried out with a ratio of TEB to $\mathrm{PPNCl}(3: 1)$ and EO in THF (v/v, $1 / 10)$ at $40{ }^{\circ} \mathrm{C}$ for $4 \mathrm{~h} .{ }^{b} \mathrm{The}$ core was prepared as in Table $1 .{ }^{c}$ Determined by GPC with THF as the eluent based on polystyrene standards. ${ }^{d}$ Measured by GPC equipped with multiangle laser light scattering (GPC-MALLS). ${ }^{e}$ The hydrolyzed linear arm blocks were analyzed by GPC calibrated with PEO standards.

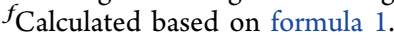

Table 4. Characterization of Amphiphilic Miktoarm Stars with Polycarbonate Cores ${ }^{a}$

\begin{tabular}{|c|c|c|c|c|c|c|c|c|c|c|}
\hline \multirow[b]{2}{*}{ entry } & \multirow{2}{*}{$\begin{array}{l}{[\mathrm{VCD}] /} \\
{\left[\mathrm{PPO}^{-}\right]}\end{array}$} & \multirow[b]{2}{*}{ time $^{b}(\mathrm{~h})$} & \multirow[b]{2}{*}{$M_{\mathrm{n} P P O}{ }^{c}(\mathrm{~kg} / \mathrm{mol})$} & \multirow[b]{2}{*}{$M_{\mathrm{n}, \mathrm{PEO}}{ }^{c}(\mathrm{~kg} / \mathrm{mol})$} & \multicolumn{2}{|c|}{ wt $\%^{d}$} & \multirow[b]{2}{*}{$M_{\mathrm{w} \mu(\mathrm{PPO})_{m}(\mathrm{PEO})_{n}}(\mathrm{~kg} / \mathrm{mol})^{e}$} & \multirow[b]{2}{*}{$M_{\mathrm{w}(\mathrm{PPO})_{m} \text { star }}(\mathrm{kg} / \mathrm{mol})^{f}$} & \multicolumn{2}{|c|}{ arm number ${ }^{g}$} \\
\hline & & & & & $\mathrm{PPO}$ & PEO & & & PPO & PEO \\
\hline 1 & 23 & 11 & 3.0 & 16.8 & 13 & 67 & 231 & 62.2 & 10.0 & 9.3 \\
\hline 2 & 23 & 17 & 4.2 & 13.0 & 24 & 52 & 255 & 122 & 14.7 & 10.0 \\
\hline 3 & 23 & 28 & 4.2 & 12.4 & 23 & 53 & 214 & 100 & 12.0 & 9.2 \\
\hline 4 & 26 & 12 & 4.2 & 14.4 & 23 & 51 & 195 & 95 & 10.8 & 6.9 \\
\hline 5 & 35 & 14 & 4.2 & 15.8 & 21 & 47 & 239 & 126 & 12.1 & 7.1 \\
\hline 6 & 35 & 18 & 4.2 & 14.4 & 21 & 48 & 258 & 134 & 12.9 & 8.6 \\
\hline 7 & 17 & 20 & 4.5 & 26.1 & 13 & 77 & 316 & 72.6 & 9.5 & 9.3 \\
\hline
\end{tabular}

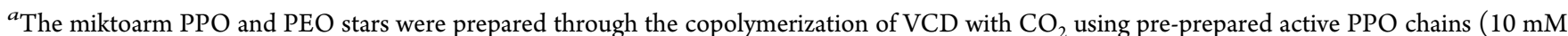
in THF) at $50^{\circ} \mathrm{C}$ and then polymerization of $\mathrm{EO}$ at $\mathrm{RT}$ through sequential addition of $\mathrm{EO}$ in THF $(\mathrm{v} / \mathrm{v}=1: 10)$ and TEB $(\mathrm{TEB} / \mathrm{PPO}-=3: 1)(\mathrm{see}$ details in the experimental part). ${ }^{b}$ Copolymerization time of $\mathrm{VCD}$ and $\mathrm{CO}_{2}$ with active PPO chains. ${ }^{c}$ Pre-prepared PPO arm and degraded PEO arm were measured by GPC with THF as the eluent based on PEO standards. ${ }^{d}$ Calculated based on the composition of PEO and PPO from NMR data of miktoarm stars, and under the assumption that complete incorporation of VCD and PPO into miktoarm stars occurred and that $80 \%$ of epoxides in VCD reacted with $\mathrm{CO}_{2} .{ }^{e}$ Measured by GPC equipped with multiangle laser light scattering (GPC-MALLS). ${ }^{f_{M}} M_{\mathrm{w}(\mathrm{PPO}))_{m} \text { star }}$ was deduced

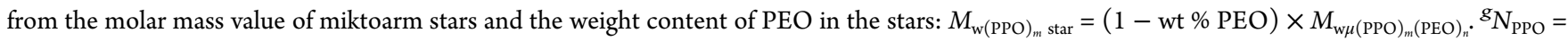
$M_{\mathrm{w} \mu(\mathrm{PPO})_{m}(\mathrm{PEO})_{n}} \times$ wt \% PPO $/ M_{\mathrm{n}, \mathrm{PPO}} ; N_{\mathrm{PEO}}=M_{\mathrm{w} \mu(\mathrm{PPO})_{m}(\mathrm{PEO})_{n}} \times$ wt $\% \mathrm{PEO} / M_{\mathrm{n}, \mathrm{PEO}}$.

375 stars carrying 5-20 arms could be prepared with a molar mass 376 of up to $835 \mathrm{~kg} / \mathrm{mol}$.

377 Synthesis of Star Block Copolymers (PVCDC)-(PPO-b${ }_{378}$ PEO) $n$ with a Degradable Polycarbonate Core. Some of the 379 polycarbonate cores formed by the core-first method were also 380 utilized to synthesize star block copolymers with arms 381 consisting of PPO- $b$-PEO chains. For this, PO was initially 382 polymerized from the "living" carbonate cores, followed by 383 polymerization of EO (Scheme 1B). Molecular character384 ization results of the star blocks prepared by this procedure are 385 shown in Table 3.

386 The distributions of GPC traces became narrower after the 387 formation of PPO stars with dispersity of molar mass around 3881.5 (Figure S2). After polymerization of EO, the GPC traces 389 shifted to the higher molar mass region and the precursor peak 390 vanished, indicating that all the PPO arms efficiently initiated 391 the ROP of EO and brought about the growth of PEO blocks. 392 The absolute molar masses of these star block copolymers 393 could be obtained by MALLS/GPC; for the $M_{n}$ values of PPO$394 b$-PEO block copolymer arms, they were determined after 395 hydrolysis of the star cores and isolation of the latter arms; 396 these values were subsequently used to determine the actual 397 number of arms in the star structure, which ranged from 5 to 398 17, values that are close to those obtained for PEO homostars. 399 Synthesis of Amphiphilic Miktoarm Stars (PVCDC)$400(P P O)_{m}(P E O)_{n}$. After the successful synthesis of PEO stars and PPO- $b$-PEO star-shaped block copolymers, we attempted 401 the synthesis of miktoarm stars $\mu-\mathrm{A}_{m} \mathrm{~B}_{n}$, composed of 402 hydrophobic poly(propylene oxide) (PPO) and hydrophilic 403 PEO arms, both of them linked to degradable polycarbonate 404 cores. The synthetic strategy adopted for the synthesis of such 405 miktoarm stars was to generate first "living" PPO arms, then 406 build degradable polycarbonate cores, and finally use the latter 407 to grow PEO arms through in- and -out sequences of reactions. 408 This methodology is similar to the one developed for the 409 synthesis of miktoarm stars via living anionic polymerization, ${ }^{53} 410$ with the difference that VCD was used as a cross-linker instead 411 of divinylbenzene to form the core.

In the in- and -out methodology, the "living" linear 413 macroinitiator PPO served to initiate the polymerization of 414 the cross-linker, VCD, and build the star core whose anionic 415 sites could then be utilized to trigger the growth of arms of a 416 second monomer, here EO (Scheme 2). Theoretically, the 417 number of initiating sites carried by the star cores must be 418 equal to the number of PPO macroinitiator arms that were 419 used to build these cores. The number of branches that could 420 be subsequently initiated and grown by polymerization of 421 another monomer, here EO, must also be more or less equal to 422 the initial number of PPO arms. However, the initiating sites 423 carried by the cores may not be all accessible to the second 424 monomer and, therefore, may vary with the extent of cross- 425 linking of the core, and the steric hindrance due to the PPO 426 
427 arms already connected to the core. As shown in the 428 subsequent section, the TEB-controlled synthetic method 429 incurs minimum purification steps and offers the major 430 advantage of being versatile enough to afford in one pot the 431 synthesis of miktoarm stars made of hydrophobic carbonate 432 cores and amphiphilic shells composed of both hydrophilic 433 PEO and hydrophobic PPO arms.

434 In the presence of $\mathrm{CO}_{2}$, "living" PPO chains with their end435 standing alkoxide active sites readily react with the latter 436 monomer and with VCD to eventually give rise to 437 polycarbonate-based cross-linked cores. As in the previously 438 described methodologies, it is vital to precisely control factors 439 such as the dilution of the reaction medium, the arm length, 440 and the amount of cross-linker utilized to avoid macroscopic 441 gelation. (PVDCD)-(PPO $)_{m}$ stars synthesized under different 442 reaction conditions (Table 4) were thus first characterized. 443 The generated carbonate cores could be easily identified by IR 444 analysis with the characteristic IR absorption due to cyclic 445 carbonates at $1808 \mathrm{~cm}^{-1}$ and acyclic carbonates at $1745 \mathrm{~cm}^{-1}$. 446 The GPC traces of these (PVCDC)-(PPO) ${ }_{m}$ stars featured a 447 broad distribution of star-shaped species (Figure 3$)$. This

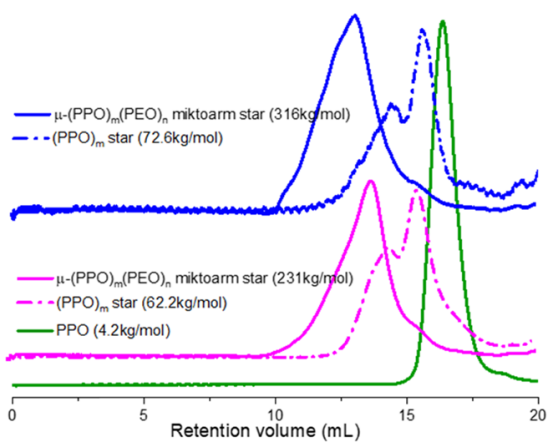

Figure 3. Representative GPC overlay profiles of linear PPO arm, PPO stars, and miktoarm $(\mathrm{PPO})_{m}(\mathrm{PEO})_{n}$ stars (lower: entry 2, upper: entry 7 in Table 4$)$.

448 shows that besides reactions contributing to the growth and to 449 the formation of each core, intermolecular star-star couplings 450 also occurred, resulting in the broad distribution of star 451 populations observed. The "bump" seen in GPC traces at 452 lower elution volumes is indicative of such star-star couplings 453 that are the products of reactions between unreacted epoxides 454 carried by a star core and the anionic sites belonging to 455 another star core. On the other hand, none of the GPC traces 456 of these (PVCDC)-(PPO) $)_{m}$ precursor stars indicates the 457 presence of residual $\mathrm{PPO}$ macroinitiator, which ascertains that 458 all of them contributed to star core formation. Unlike the case 459 of the core-first methodology, where intermolecular star-star 460 coupling could occur without major interference due to steric 461 hindrance and, thus, could give rise to rather homogeneous 462 cores, the arm-first methodology did not afford a unimodal 463 distribution of star-shaped species because the star-star 464 coupling phenomenon could take place only partially due to 465 the hindrance by the PPO arms. Typically and whatever the 466 experimental conditions used, we observed a bimodal 467 distribution of star-shaped species: the first population at 468 higher elution volumes corresponds to stars obtained directly 469 by the reaction of "living" PPO macroinitiators with VCD and $470 \mathrm{CO}_{2}$, and the second at lower elution volumes corresponds to 471 star-star couplings. Upon increasing the reaction time, this 472 second population of star-shaped species grew at the expense of the first population. Augmenting the concentration of cross- 473 linker to values higher than those indicated in Table 4474 generally resulted in the macrogelation of the medium within 475 the dilution generally utilized. Using PPO macroinitiators of 476 small size was beneficial to the formation of homogeneous star- 477 shaped species. It was not attempted to determine the actual 478 molar masses of the star formed and to deduce their number of 479 arms at this stage. It was only after the growth of PEO arms in 480 the subsequent step that the actual molar masses of miktoarm 481 stars were measured and the number of PEO arms and that of 482 PPO arms were deduced.

483

In a third step, EO was added to grow PEO arms from these 484 active sites carried by the cores to generate miktoarm stars. 485 Upon characterization of the samples eventually obtained by 486 ${ }^{1} \mathrm{H}$ NMR (Figure S3), a new highly intense band 487 corresponding to protons of $\mathrm{CH}_{2} \mathrm{CH}_{2} \mathrm{O}$ linkages was visible 488 at $3.5 \mathrm{ppm}$. The molar mass distributions of the obtained 489 miktoarm stars with their polycarbonate cores all became 490 narrower, although slightly broad (Figure 3). To be sure that 491 no linear contaminants are present in the miktoarm star 492 samples, PPO- $b$-PCHC- $b$-PEO triblock terpolymers of approx- 493 imately the same composition as that of miktoarm stars were 494 synthesized by sequential polymerization of $\mathrm{PO}, \mathrm{CHO}$, and 495 EO. Both miktoarm stars and the latter triblock copolymers 496 were characterized by dynamic light scattering (DLS), and the 497 respective distributions of hydrodynamic sizes were compared. 498 Miktoarm polymers exhibit a hydrodynamic size very different 499 from that of the linear block copolymers $(d$ size $<2 \mathrm{~nm})$ even 500 after stirring the samples for five days in DMF solvent, 501 confirming the absence of any linear block copolymer 502 contaminant in the miktoarm star samples (Figure S4). The 503 absolute molar masses of miktoarm stars were then determined 504 by light scattering. Given the compositions drawn from the 505 ratio of intensities of NMR peaks due to PPO and PEO blocks 506 in the miktoarm star samples (Figure S3) and the feeding 507 amount of VCD and PPO leading to the formation of 508 (PVCDC)-(PPO $)_{m}$ stars, the weight fraction of the PEO arms 509 and PPO arms in the miktoarm stars could be obtained, and 510 thus, the molar masses of the PPO star precursors could be 511 deduced. As shown in Table 4, based on the molar mass of 512 PEO arms obtained after hydrolysis of the miktoarm star cores 513 and that of PPO arms present, the average number of PEO 514 arms and PPO arms could be respectively calculated. The 515 presence of slightly more PPO arms than PEO arms in the 516 formed miktoarm stars indicates that some initiating sites were 517 inaccessible to the second monomer.

518

Hydrolysis of the Star Polycarbonate Cores. The 519 primary objective of this study was to impart degradability to 520 the star cores. Toward this goal, hydrolysis was attempted 521 under basic conditions at $50{ }^{\circ} \mathrm{C}$. The adopted reaction 522 conditions facilitated the degradation of the highly branched 523 core, which is composed of carbonate units, releasing PPO and 524 PEO arms. The product of degradation was then extracted 525 with DCM to isolate PEO and PPO arms, which were further 526 characterized using FT-IR, NMR, GPC, and MALDI-TOF MS. 527

Characteristic IR absorptions of linear acyclic carbonates 528 and cyclic carbonates ( 1745 and $1808 \mathrm{~cm}^{-1}$ ) were absent in 529 the hydrolyzed sample (Figure S5). Besides, the ${ }^{1} \mathrm{H}$ NMR 530 spectrum of the hydrolyzed star sample, (PVCDC)-(PEO $)_{n}, 531$ after precipitation showed peaks solely of the PEO chains (3.5 532 $\mathrm{ppm}$ ) and was devoid of peaks of any carbonate linkages from 533 the core networks, thus confirming the degradation of 534 carbonate cores within the star structure (Figure S6). This 535 
536 implies the effectiveness of polycarbonate as both a macro537 anionic initiator and a degradable core network. These claims 538 were further corroborated by the GPC analysis of the 539 hydrolysis products (in THF using PEO standard) and 540 MALDI-TOF MS characterization. It was observed that 541 homo-PEO stars display a unimodal distribution correspond542 ing to their unhydrolyzed PEG arm chains. Figure 4A reveals
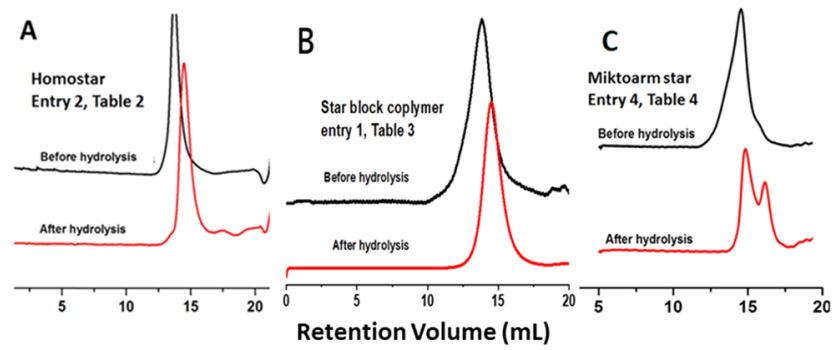

Figure 4. Representative GPC traces of hydrolyzed samples.

543 the shift of GPC traces of star polymers after hydrolytic 544 degradation, clearly indicative of chain scissions within the 545 carbonate cross-linked networks, which freed PEO arms. 546 Degraded star-shaped block copolymers also exhibited a 547 unimodal distribution arising from their unhydrolyzed PPO$548 b$-PEO arms (Figure 4B). On the other hand, miktoarm stars 549 produced upon hydrolysis a bimodal distribution of polymer 550 populations and, thus, revealed the presence of two separate 551 peaks by GPC analysis corresponding to each type of arm 552 species PEO and PPO (Figure 4C). In these amphiphilic stars, 553 apart from PPO and PEO segments, all other moieties consist 554 of carbonate units, which were degraded by hydrolysis. This 555 was further confirmed by the NMR spectrum of degraded star556 shaped block copolymers and miktoarm stars (Figures S7 and 557 S8).

558 In MALDI-TOF MS (Figure S9) of the hydrolyzed samples, 559 populations of peaks with a difference of $\mathrm{m} / z 44$ were 560 observed, which corresponds to hydrolyzed PEO chains 561 separated from the core. Notably, it can be clearly seen that 562 the main population corresponds to PEO chains terminated 563 with hydroxyls at both ends, where two other populations 564 corresponding to PEO chains terminated at one end by 565 hydroxyl groups and the other end by VCD derivatives also 566 exist. All of these positive observations strongly substantiate 567 the formation of star polymers with fully degradable cores.

\section{CONCLUSIONS}

569 The advantages of star polymers that contain hydrolyzable 570 cores are manifold for a wide range of applications for PEG571 based drug delivery systems. Taking advantage of the 572 chemistry of TEB-activated anionic copolymerization of 573 epoxide with $\mathrm{CO}_{2}$, we succeeded in incorporating degradable 574 carbonate units within the core structure of star polymers. 575 Polycarbonate macroanions with $80-90 \%$ carbonate content 576 were synthesized by a series of reactions through the "core577 first" and "arm-first" methodologies and were further used to 578 grow EO arms. These star polymers are proven to be 579 susceptible to selective degradation of their core, allowing 580 the isolation of arm chains. Degradation experiments were 581 carried out under basic conditions, but they could have been 582 undertaken under acidic conditions $(\mathrm{pH}<5)$ as carbonate 583 linkages are known to undergo degradation under both basic 584 and acidic conditions. This boron-based chemistry is versatile enough not only to build hydrolyzable polycarbonate cores but 585 also to grow polyether arms without affecting the integrity of 586 carbonate linkages. The same chemistry could also be 587 successfully used to assemble other types of hydrolyzable 588 stars, including polystyrene and polydiene stars, through the 589 core-first methodology.

\section{ASSOCIATED CONTENT}

590

SI Supporting Information

591

The Supporting Information is available free of charge at 593 https://pubs.acs.org/doi/10.1021/acs.macromol.9b02658. 594

Characterization data of IR, NMR, GPC, DLS, and 595 MALDI-TOF (PDF)

\section{AUTHOR INFORMATION}

Corresponding Authors

Yves Gnanou - Physical Sciences and Engineering Division, King 599 Abdullah University of Science and Technology (KAUST), 600 Thuwal 23955, Saudi Arabia; (1) orcid.org/0000-0001-6253- 601 7856; Email: yves.gnanou@kaust.edu.sa 602

Xiaoshuang Feng - Physical Sciences and Engineering Division, 603 King Abdullah University of Science and Technology (KAUST), 604 Thuwal 23955, Saudi Arabia; $\odot$ orcid.org/0000-0001-7473- 605 1728; Email: fxs101@gmail.com

Authors 607

Dhanya Augustine - Physical Sciences and Engineering 608 Division, King Abdullah University of Science and Technology 609 (KAUST), Thuwal 23955, Saudi Arabia

610

Nikos Hadjichristidis - KAUST Catalysis Center, Physical 611 Sciences and Engineering Division, King Abdullah University of 612 Science and Technology (KAUST), Thuwal 23955, Saudi 613 Arabia; (1) orcid.org/0000-0003-1442-1714

Complete contact information is available at:

https://pubs.acs.org/10.1021/acs.macromol.9b02658

614

Notes

The authors declare no competing financial interest.

\section{ACKNOWLEDGMENTS}

618

This research work was supported by KAUST under baseline 620 funding (BAS/1/1374-01-01).

\section{REFERENCES}

622

(1) D'souza, A. A.; Shegokar, R. Polyethylene glycol (PEG): a 623 versatile polymer for pharmaceutical applications. Expert Opin. Drug 624 Delivery 2016, 13, 1257-1275.

(2) Suk, J. S.; Xu, Q.; Kim, N.; Hanes, J.; Ensign, L. M. PEGylation 626 as a strategy for improving nanoparticle-based drug and gene delivery. 627 Adv. Drug Delivery Rev. 2016, 99, 28-51.

(3) Pasut, G.; Veronese, F. M. State of the art in PEGylation: The 629 great versatility achieved after forty years of research. J. Controlled 630 Release 2012, 161, 461-472.

631

(4) Knop, K.; Hoogenboom, R.; Fischer, D.; Schubert, U. S. 632 Poly(ethylene glycol) in Drug Delivery: Pros and Cons as Well as 633 Potential Alternatives. Angew. Chem., Int. Ed. 2010, 49, 6288-6308. 634

(5) Harris, J. M.; Chess, R. B. Effect of pegylation on 635 pharmaceuticals. Nat. Rev. Drug Discovery 2003, 2, 214-221. 636

(6) Herzberger, J.; Niederer, K.; Pohlit, H.; Seiwert, J.; Worm, M.; 637 Wurm, F. R.; Frey, H. Polymerization of ethylene oxide, propylene 638 oxide, and other alkylene oxides: Synthesis, novel polymer 639 architectures, and bioconjugation. Chem. Rev. 2016, 116, 2170-2243. 640

(7) Abbina, S.; Vappala, S.; Kumar, P.; Siren, E. M. J.; La, C. C.; 641 Abbasi, U.; Brooks, D. E.; Kizhakkedathu, J. N. Hyperbranched 642 
643 polyglycerols: recent advances in synthesis, biocompatibility and 644 biomedical applications. J. Mater. Chem. B 2017, 5, 9249-9277.

645 (8) Lapienis, G. Star-shaped polymers having PEO arms. Prog. 646 Polym. Sci. 2009, 34, 852-892.

647 (9) Taton, D.; Feng, X. S.; Gnanou, Y. Dendrimer-like polymers: a 648 new class of structurally precise dendrimers with macromolecular 649 generations. New J. Chem. 2007, 31, 1097-1110.

650 (10) Hawker, C. J.; Chu, F.; Pomery, P. J.; Hill, D. J. T. 651 Hyperbranched Poly(ethylene glycol)s: A New Class of Ion652 Conducting Materials. Macromolecules 1996, 29, 3831-3838.

653 (11) Thomas, A.; Müller, S. S.; Frey, H. Beyond poly(ethylene 654 glycol): Linear polyglycerol as a multifunctional polyether for 655 biomedical and pharmaceutical applications. Biomacromolecules 656 2014, 15, 1935-1954.

657 (12) Perevyazko, I.; Seiwert, J.; Schömer, M.; Frey, H.; Schubert, U. 658 S.; Pavlov, G. M. Hyperbranched Poly(ethylene glycol) Copolymers: 659 Absolute Values of the Molar Mass, Properties in Dilute Solution, and 660 Hydrodynamic Homology. Macromolecules 2015, 48, 5887-5898.

661 (13) Zhu, S.; Xia, R.; Chen, P.; Yang, B.; Miao, J.; Zheng, Z.; Su, L.; 662 Qian, J.; Cao, M.; Feng, X. High-efficiency synthesis of dendrimer-like 663 poly (ethylene oxide) via "arm-first" approach. J. Polym. Res. 2017, 24, $6641-10$.

665 (14) Feng, X.; Chaikof, E. L.; Absalon, C.; Drummond, C.; Taton, 666 D.; Gnanou, Y. Dendritic carrier based on PEG: Design and 667 degradation of acid-sensitive dendrimer-like poly(ethylene oxide)s. 668 Macromol. Rapid Commun. 2011, 32, 1722-1728.

669 (15) Feng, X. S.; Taton, D.; Chaikof, E. L.; Gnanou, Y. Fast Access 670 to Dendrimer-like Poly(ethylene oxide)s through Anionic Ring671 Opening Polymerization of Ethylene Oxide and Use of Nonprotected 672 Glycidol as Branching Agent. Macromolecules 2009, 42, 7292-7298. 673 (16) Feng, X.; Taton, D.; Ibarboure, E.; Chaikof, E. L.; Gnanou, Y. 674 Janus-type dendrimer-like poly(ethylene oxide)s. J. Am. Chem. Soc. $6752008,130,11662-11676$.

676 (17) Feng, X.; Taton, D.; Borsali, R.; Chaikof, E. L.; Gnanou, Y. pH 677 responsiveness of dendrimer-like poly(ethylene oxide)s. J. Am. Chem. 678 Soc. 2006, 128, 11551-11562.

679 (18) Feng, X. S.; Taton, D.; Chaikof, E. L.; Gnanou, Y. Toward an 680 easy access to dendrimer-like poly(ethylene oxide)s. J. Am. Chem. Soc. 681 2005, 127, 10956-10966.

682 (19) Zheng, K.; Ren, J.; He, J. Thermally Responsive Unimolecular 683 Nanoreactors from Amphiphilic Dendrimer-Like Copolymer Pre684 pared via Anionic Polymerization and Cross Metathesis Reaction. 685 Macromolecules 2019, 52, 6780-6791.

686 (20) Walach, W.; Trzebicka, B.; Justynska, J.; Dworak, A. High 687 molecular arborescent polyoxyethylene with hydroxyl containing shell. 688 Polymer 2004, 45, 1755-1762.

689 (21) Lapienis, G.; Penczek, S. One-pot synthesis of star-shaped 690 macromolecules containing polyglycidol and poly(ethylene oxide) 691 arms. Biomacromolecules 2005, 6, 752-762.

692 (22) Lapienis, G.; Penczek, S. Reaction of Oligoalcohols with 693 Diepoxides: An Easy, One-Pot Way to Star-Shaped, Multibranched 694 Polymers. II. Poly(ethylene oxide) Stars - Synthesis and Analysis by 695 Size Exclusion Chromatography Triple-Detection Method. J. Polym. 696 Sci., Part A: Polym. Chem. 2004, 42, 1576-1598.

697 (23) Lapienis, G.; Penczek, S. Highly branched (starlike) polymers 698 obtained by reacting oligoalcohols with dicyclic compounds. 1 . 699 Monomethoxy poly(ethylene oxide) and diepoxides. Macromolecules 700 2000, 33, 6630-6632.

701 (24) Hou, S.; Taton, D.; Saule, M.; Logan, J.; Chaikof, E. L.; 702 Gnanou, Y. Synthesis of functionalized multiarm poly(ethylene oxide) 703 stars. Polymer 2003, 44, 5067-5074.

704 (25) Taton, D.; Saule, M.; Logan, J.; Duran, R.; Hou, S.; Chaikof, E. 705 L.; Gnanou, Y. Polymerization of ethylene oxide with a calixarene706 based precursor: Synthesis of eight-arm poly(ethylene oxide) stars by 707 the core-first methodology. J. Polym. Sci., Part A: Polym. Chem. 2003, 708 41, 1669-1676.

709 (26) Comanita, B.; N, B.; Roovers, J. Star Poly(ethylene oxide)s 710 from Carbosilane Dendrimers. Macromolecules 1999, 32, 1069-1072.
(27) Knischka, R.; Lutz, P. J.; Sunder, A.; Mülhaupt, R.; Frey, H. 711 Functional Poly(ethylene oxide) Multiarm Star Polymers: Core-First 712 Synthesis Using Hyperbranched Polyglycerol Initiators. Macro- 713 molecules 2000, 33, 315-320.

(28) Rein, D.; Lamps, J. P.; Rempp, P.; Lutz, P.; Papanagopoulos, 715 D.; Tsitsilianis, C. New developments in synthesis of star polymers 716 with poly(ethylene oxide) arms. Acta Polym. 1993, 44, 225-229. 717

(29) Gnanou, Y.; Lutz, P.; Rempp, P. Synthesis of star-shaped 718 poly(ethylene oxide). Makromol. Chem. 1988, 189, 2885-2892. 719

(30) Shibuya, Y.; Tatara, R.; Jiang, Y.; Shao-Horn, Y.; Johnson, J. A. 720 Brush-First ROMP of poly(ethylene oxide) macromonomers of varied 721 length: impact of polymer architecture on thermal behavior and $\mathrm{Li}+722$ conductivity. J. Polym. Sci., Part A: Polym. Chem. 2019, 57, 448-455. 723 (31) Jin, X.; Sun, P.; Tong, G.; Zhu, X. Star polymer-based 724 unimolecular micelles and their application in bio-imaging and 725 diagnosis. Biomaterials 2018, 178, 738-750. 726

(32) Ren, J. M.; McKenzie, T. G.; Fu, Q.; Wong, E. H. H.; Xu, J.; 727 An, Z.; Shanmugam, S.; Davis, T. P.; Boyer, C.; Qiao, G. G. Star 728 Polymers. Chem. Rev. 2016, 116, 6743-6836.

(33) Wu, W.; Wang, W.; Li, J. Star polymers: Advances in 730 biomedical applications. Prog. Polym. Sci. 2015, 46, 55-85. 731

(34) Webster, R.; Didier, E.; Harris, P.; Siegel, N.; Stadler, J.; 732 Tilbury, L.; Smith, D. PEGylated Proteins: Evaluation of Their Safety 733 in the Absence of Definitive Metabolism Studies. Drug Metab. Dispos. 734 2007, 35, 9-16.

(35) Varghese, J. K.; Hadjichristidis, N.; Gnanou, Y.; Feng, X.736 Degradable poly (ethylene oxide) through metal-free copolymerization 737 of ethylene oxide with l-lactide. Polym. Chem. 2019, 10, 3764-3771. 738 (36) Dingels, C.; Muller, S. S.; Steinbach, T.; Tonhauser, C.; Frey, 739 $\mathrm{H}$. Universal Concept for the Implementation of a Single Cleavable 740 Unit at Tunable Position in Functional Poly(ethylene glycol)s. 741 Biomacromolecules 2013, 14, 448-459.

(37) Tonhauser, C.; Schüll, C.; Dingels, C.; Frey, H. Branched acid- 743 degradable, biocompatible polyether copolymers via anionic ring- 744 opening polymerization using an epoxide inimer. ACS Macro Lett. 745 2012, 1, 1094-1097.

(38) Cho, H. Y.; Srinivasan, A.; Hong, J.; Hsu, E.; Liu, S.; Shrivats, 747 A.; Kwak, D.; Bohaty, A. K.; Paik, H.-j.; Hollinger, J. O.; 748 Matyjaszewski, K. Synthesis of Biocompatible PEG-Based Star 749 Polymers with Cationic and Degradable Core for siRNA Delivery. 750 Biomacromolecules 2011, 12, 3478-3486.

751

(39) Cuthbert, J.; Yerneni, S. S.; Sun, M.; Fu, T.; Matyjaszewski, K. 752 Degradable Polymer Stars Based on Tannic Acid Cores by ATRP. 753 Polymers 2019, 11, 752.

(40) Liu, J.; Burts, A. O.; Li, Y.; Zhukhovitskiy, A. V.; Ottaviani, M. 755 F.; Turro, N. J.; Johnson, J. A. "Brush-first" method for the parallel 756 synthesis of photocleavable, nitroxide-labeled poly (ethylene glycol) 757 star polymers. J. Am. Chem. Soc. 2012, 134, 16337-16344. 758 (41) Zhang, D. D.; Feng, X. S.; Gnanou, Y.; Huang, K. W. 759 Theoretical Mechanistic Investigation into Metal-Free Alternating 760 Copolymerization of $\mathrm{CO} 2$ and Epoxides: The Key Role of 761 Triethylborane. Macromolecules 2018, 51, 5600-5607. 762

(42) Zhang, D.; Boopathi, S. K.; Hadjichristidis, N.; Gnanou, Y.; 763 Feng, X. Metal-Free Alternating Copolymerization of CO2 with 764 Epoxides: Fulfilling "Green" Synthesis and Activity. J. Am. Chem. Soc. 765 2016, 138, 11117-11120.

(43) Chen, Y.; Shen, J.; Liu, S.; Zhao, J.; Wang, Y.; Zhang, G. High 767 Efficiency Organic Lewis Pair Catalyst for Ring-Opening Polymer- 768 ization of Epoxides with Chemoselectivity. Macromolecules 2018, 51, 769 8286-8297.

(44) Zhang, C.-J.; Duan, H.-Y.; Hu, L.-F.; Zhang, C.-H.; Zhang, X.- 771 H. Metal-Free Route to Precise Synthesis of Poly(propylene oxide) 772 and Its Blocks with High Activity. ChemSusChem 2018, 11, 4209- 773 4213.

(45) Boopathi, S. K.; Hadjichristidis, N.; Gnanou, Y.; Feng, X. Direct 775 access to poly (glycidyl azide) and its copolymers through anionic (co- 776 )polymerization of glycidyl azide. Nat. Commun. 2019, 10, No. 293. 777

(46) Zhao, J.; Pahovnik, D.; Gnanou, Y.; Hadjichristidis, N. 778 Phosphazene-promoted metal-free ring-opening polymerization of 779 
780 ethylene oxide initiated by carboxylic acid. Macromolecules 2014, 47, 781 1693-1698.

782 (47) Zhang, Z.; Kuijer, R.; Bulstra, S. K.; Grijpma, D. W.; Feijen, J. 783 The in vivo and in vitro degradation behavior of poly (trimethylene 784 carbonate). Biomaterials 2006, 27, 1741-1748.

785 (48) Tangpasuthadol, V.; Pendharkar, S. M.; Peterson, R. C.; Kohn, $786 \mathrm{~J}$. Hydrolytic degradation of tyrosine-derived polycarbonates, a class 787 of new biomaterials. Part II: 3-yr study of polymeric devices. 788 Biomaterials 2000, 21, 2379-2387.

789 (49) Meabe, L.; Sardon, H.; Mecerreyes, D. Hydrolytically 790 degradable poly(ethylene glycol) based polycarbonates by organo791 catalyzed condensation. Eur. Polym. J. 2017, 95, 737-745.

792 (50) Stoll, G. H.; Nimmerfall, F.; Acemoglu, M.; Bodmer, D.; Bantle, 793 S.; Müller, I.; Mahl, A.; Kolopp, M.; Tullberg, K. Poly(ethylene 794 carbonate)s, part II1: degradation mechanisms and parenteral delivery 795 of bioactive agents. J. Controlled Release 2001, 76, 209-225.

796 (51) Khanna, K.; Varshney, S.; Kakkar, A. Miktoarm star polymers: 797 Advances in synthesis, self-assembly, and applications. Polym. Chem. 798 2010, 1, 1171-1185.

799 (52) Han, B.; Zhang, L.; Zhang, H.; Ding, H.; Liu, B.; Wang, X. 800 One-pot synthesis and postpolymerization functionalization of cyclic 801 carbonate/epoxide-difunctional polycarbonates prepared by regiose802 lective diepoxide/CO2 copolymerization. Polym. Chem. 2016, 7, $8034453-4457$.

804 (53) Iatrou, H.; Avgeropoulos, A.; Sakellariou, G.; Pitsikalis, M.; 805 Hadjichristidis, N. CHAPTER 1 Miktoarm Star ( $\mu$-Star) Polymers: A 806 Successful Story. In Miktoarm Star Polymers: From Basics of Branched 807 Architecture to Synthesis, Self-assembly and Applications; The Royal 808 Society of Chemistry, 2017; pp 1-30. 